\title{
Recovery of an Injured Corticospinal Tract in a Patient With Infarction on the Corona Radiata
}

\author{
Sook Lee', Hyoung-Won Lim², Sang-Seok Yeo² \\ 'Department of Nursing, College of Health Sciences, Dankook University; ${ }^{2}$ Department of Physical Therapy, College of Health Sciences, Dankook \\ University, Cheonan, Chungcheongnam-do, Korea
}

Purpose: Many studies have attempted to elucidate the motor recovery mechanisms of stroke. In the current study, we report on a patient with infarction who showed recovery of an injured corticospinal tract (CST), using diffusion tensor imaging (DTI).

Methods: A 20-year-old male presented with severe paralysis of the left extremities following an infarction in the right corona radiate and basal ganglia. The patient showed good motor recovery as much as being able to manipulate object using his left hand and could walk independently on stairs at 9 months after onset.

Results: At 1 month after onset, the right CST showed decreased fiber volume due to the partial injury at the level of the corona radiate, and no (motor evoked potential) MEP was evoked from the right hemisphere. By contrast, the results at 9 months showed increased fiber volume of the right CST and the amplitude of MEP had improved to normal range on TMS.

Conclusion: These results suggest that the severely injured right CST and MEP amplitude had been recovered during 9 months. Therefore, this result has important implications for brain plasticity and brain rehabilitation in patients with infarction.

Keywords: Stroke, Corticospinal tract, Motor recovery

\section{INTRODUCTION}

Motor weakness is one of the most serious disabling sequelae of stroke. ${ }^{1,2}$ Elucidation of the mechanisms of motor recovery in stroke is important because such information could provide scientific information for stroke rehabilitation and for prediction of prognosis. Therefore, many studies have attempted to find the motor recovery mechanisms in stroke, and the contribution of the CST has been reported as a motor recovery mechanism. ${ }^{1-3}$

With regard to the motor function, the corticospinal tract (CST) is the major motor tract that mediates voluntary movements in the human brain. ${ }^{3}$ In addition, The CST is known to be primarily involved in fine motor skills, such as hand function. A number of studies have reported on stroke patients who were unable to perform fine motor activities of the hands after complete injury of the lateral CST. ${ }^{1-3}$ Recent advances in diffusion tensor imaging (DTI)

Received January 21, 2015 Received February 9, 2015

Accepted February 11, 2015

Corresponding author Sang-Seok Yeo

E-mail yeopt@dankook.ac.kr

- This work was supported by the Rural Development Administration. have enabled investigators to estimate the state of the CST at the subcortical level. ${ }^{4,5}$ Many studies have demonstrated the usefulness of DTI in estimating the state of the CST in patients with stroke. ${ }^{6-10}$ However, very little is known about the recovery of injured CST in patients with infarction.

In the current study, we attempted to investigate the recovery of injured CST in patient with infarction on the cororna radiate, using DTI.

\section{METHODS}

\section{Subjects}

A 20-year-old male presented with severe paralysis of the left upper and lower extremities, which occurred at the onset of infarction (Motricity index [MI]: 30 [full mark: 100] ]11. T2-weighted MR images showed infarction in the right corona radiate and basal ganglia at

Copylight ( 2015 The Korean Society of Physical Therapy

This is an Open Access article distribute under the terms of the Creative Commons Attribution Non-commercial License (Http:// creativecommons.org/license/by-nc/3.o.) which permits unrestricted non-commercial use, distribution, and reproduction in any medium, provided the original work is properly cited. 
1 month after onset (Fig. 1A). He received comprehensive rehabilitative management, including, physical therapy, and neuromuscular electrical stimulation of the affected finger extensors and ankle dorsiflexors. Physical therapy focused on improvement of motor function of the left upper and lower extremities and was performed five times per week. Weakness of his left extremities improved from the MI score of 30 points at onset to 93 points ( 9 months) during 8 months period of rehabilitation. As a result, he was able to extend the affected fingers against gravity at 9 months after onset and could walk independently on even stair. The patient signed an informed consent statement, and the study protocol was approved by our institutional review board.

\section{Experimental methods}

\section{1) Diffusion tensor tractography}

Diffusion tensor images were acquired with a synergy-L Sensitivity
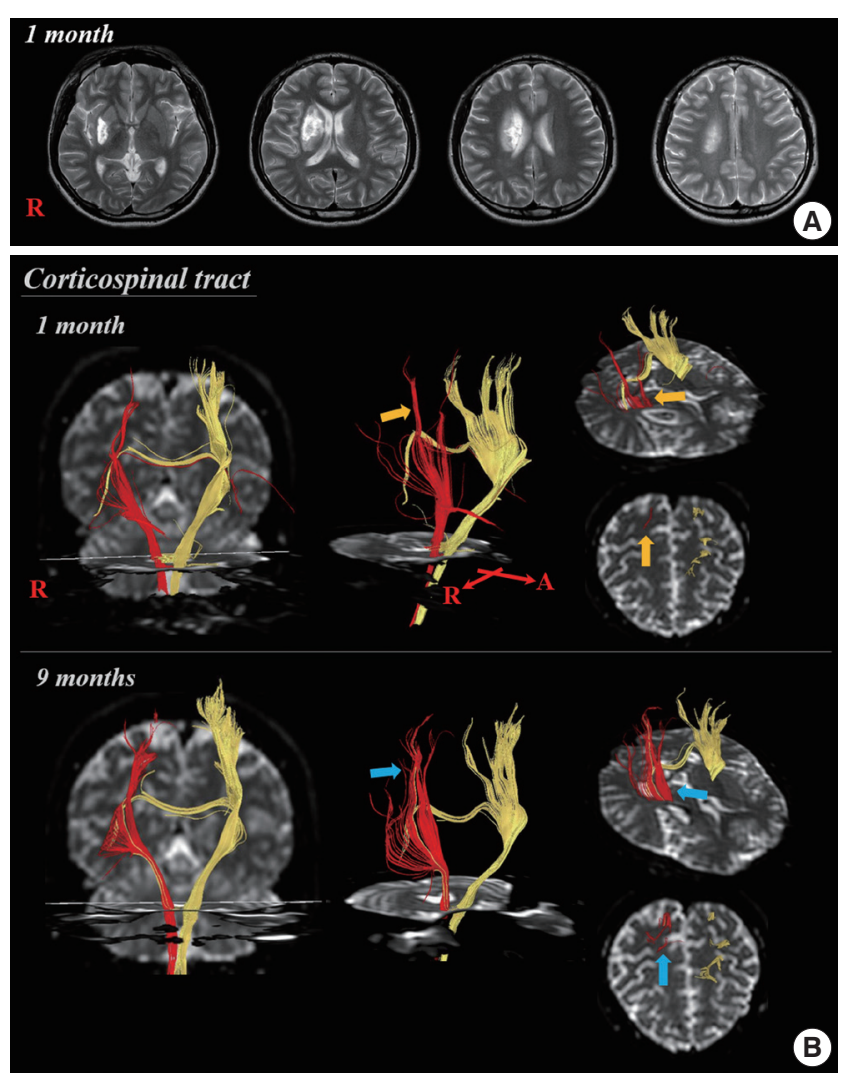

Figure 1. (A) T2-weighted MR images showed a infarction in the right corona radiata including basal ganglia at 1 month after onset. (B) Results of diffusion tensor imaging for the corticospinal tract (CST). The 1 -month DTI results showed that fiber volume of the right CST was decreased at the level of corona radiate (arrow-1 month). By contrast, the 9-month DTI results showed nearly normal level of fiber volume in the right CST (arrow-9 months).
Encoding (SENSE) head coil on a 1.5-T Philips Gyroscan Intera system using a single-shot EPI with a navigator echo. For each of the 32 noncollinear diffusion-sensitizing gradients, we acquired 60 contiguous slices $($ matrix $=192 \times 192, F O V=240 \mathrm{~mm}, \mathrm{TR} / \mathrm{TE}=10,726 /$ $76 \mathrm{~ms}, \mathrm{~b}=600 \mathrm{~mm}^{2} \mathrm{~s}^{-1}, \mathrm{NEX}=1$, thickness $=2.5 \mathrm{~mm}$ ). Three-dimensional reconstructions of fiber tracts were obtained using the DTI task card software (Philips Extended MR Work Space 2.6.3) (threshold fractional anisotropy $(\mathrm{FA})=0.15$, angle $=45^{\circ}$ ). Fiber tracts passing through both region of interests (CST portion of anterior mid-pons and low-pons on the color map) were designated as the final tracts of interest. . $^{12-14}$

\section{2) Transcranial magnetic stimulation}

TMS was performed using a Magstim Novametrix 200 magnetic stimulator with a 9-cm mean diameter circular coil (Novametrix Inc). Cortical stimulation was performed with the coil held tangentially over the vertex. The left hemisphere was stimulated by a counterclockwise current and the right hemisphere was stimulated by a clockwise current. Motor-evoked potentials (MEPs) were obtained from both abductor pollicis brevis muscles in a relaxed state. The excitatory threshold (ET) was defined as the minimum stimulus required to elicit an MEP with a peak-to-peak amplitude of $50 \mathrm{uV}$ or greater, in two out of four attempts. Stimulation intensity was set at the ET plus $20 \%$ of the maximum stimulator output. Each site was stimulated three times at intervals with a minimum of 10 seconds, from which the shortest latency and the average peak to peak amplitudes were adopted.

\section{RESULTS}

The 1-month and 9-month DTI results showed that the right CST was originated from the cerebral cortex including the M1 passed through the known CST pathway. In the left (unaffected) hemisphere, the 1 month DTT of the CST was originated from the left M1 and descended along the CST pathway, however, right CST showed decreased fiber volume compared with that of left hemisphere. On the other hand, the 9 months DTT of right CST showed increased fiber volume.

No MEP was evoked from the right hemisphere even though the stimulation intensity was increased to $100 \%$ of maximal output at 1 month. However, the amplitude was increased to a nearly normal 
range at 9-month TMS study (latency: $22.0 \mathrm{msec}$, amplitude: 1.9 mV, ET: 90\%).

\section{DISCUSSION}

In the current study, we found that this patient showed good motor recovery of left hemiplegia from infarction on the right corona radiata onset to 9 months MI, onset- 30 points (maximum: 100 point) and 9 months - 93 point. It appears that the partial injury of right CST recovered between 1 month and 9 months by the following evidence: first, the 1 month DTT showed that fiber volume of the right CST was decreased at the level of corona radiate around the infarction site. However, the fiber volume of the right CST was found to be recovered between the right primary motor cortex and the infarction site on the 9 months DTT. TMS also showed compatible results with the changes of DTT. No MEP was evoked from the affected (left) hand, even when stimulation of the affected motor cortex was at a maximal level with a round stimulator at the 1 month after onset. However, amplitude of MEP improved to normal range on TMS at the 9 months after onset. Consequently, increased fiber volumes of the CST in the affected hemisphere appear to be related to recovery of motor functions and walking ability in a patient.

Many DTI studies have reported on the recovery of injured CST at the subcortical level in $\mathrm{ICH}^{7-9,15}$ In 2006, Jang et al. demonstrated that a patient with an intracerebral hemorrhage $(\mathrm{ICH})$ who showed recovery of a partially damaged CST in terms of changes in DTI parameters and DTT on the lesion. ${ }^{7}$ Subsequently, Jang et al. ${ }^{8}$ reported on the recovery process of the damaged CST in a patient with a subcortical ICH using combined examination with DTT, TMS and functional MRI. In 2008, Yang et al. ${ }^{9}$ reported on a patient with ICH in the left corona radiata who showed recovery of a severely damaged CST using DTT and TMS. The CST of the affected hemisphere was discontinued at ICH on 3-week DTT; however, the discontinuation of the affected CST was recovered on 10-month DTT with motor recovery. Recently, Kwon et al. ${ }^{15}$ demonstrated that a patient with ICH who showed recovery of severely injured CST between 6 weeks and 12 weeks after ICH onset, using DTI. However, there was no study which reported the recovery of injured CST in a patient with infarction on the corona radiate.

In conclusion, we described a patient with infarction who showed good recovery of injured CST. To our knowledge, this is the first study to demonstrate the recovery of the injured CST by the increment of fiber volume in a patient with infarction. Therefore, this result has important implications for brain plasticity and brain rehabilitation in patients with infraction. However, this study is limited by case report. Further complementary studies involving larger case numbers are warranted.

\section{REFERENCES}

1. Jorgensen HS, Nakayama H, Raaschou HO, et al. Outcome and time course of recovery in stroke. Part II: Time course of recovery. The Copenhagen Stroke Study. Arch Phys Med Rehabil. 1995;76(5):406-412.

2. Kwelly-Hayes M, Wolf PA, Kase CS, et al. Time course of functional recovery after stroke: The framingham study. Neurorehab Neural Re. 1989; 3(2):65-70.

3. Jang $\mathrm{SH}$. The role of the corticospinal tract in motor recovery in patients with a stroke: A review. NeuroRehabilitation. 2009;24(3):285-90.

4. Mori S, van Zijl PC. Fiber tracking: Principles and strategies - a technical review. NMR Biomed. 2002;15(7-8):468-80.

5. Kwon HI, Hong JH, Kwon YH, et al. Neural tract injuries by penetration of foreign body: A diffusion tensor tractography study. J Kor Phys Ther. 2013;25(3):132-5.

6. Jang WH, Lee MY, Kwon HY, et al. Transcallosal ipsilateral motor pathway from the unaffected hemisphere in a patient with traumatic brain injury. J Kor Phys Ther. 2014;26(3):216-9.

7. Jang SH, Byun WM, Han BS, et al. Recovery of a partially damaged corticospinal tract in a patient with intracerebral hemorrhage: A diffusion tensor image study. Restor Neurol Neurosci. 2006;24(1):25-9.

8. Jang SH, Kim SH, Cho SH, et al. Demonstration of motor recovery process in a patient with intracerebral hemorrhage. NeuroRehabilitation. 2007;22(2):141-5.

9. Yang DS, Kim DS, Kim YH, et al. Demonstration of recovery of a severely damaged corticospinal tract: A diffusion tensor tractography and transcranial magnetic stimulation follow-up study. J Comput Assist Tomogr. 2008;32(3):418-20.

10. Basser PJ, Pierpaoli C. Microstructural and physiological features of tissues elucidated by quantitative-diffusion-tensor MRI. J Magn Reson B. 1996;111(3):209-19.

11. Demeurisse G, Demol O, Robaye E. Motor evaluation in vascular hemiplegia. Eur Neurol. 1980;19(6):382-9.

12. Jang SH. Somatotopic arrangement and location of the corticospinal tract in the brainstem of the human brain. Yonsei Med J. 2011;52(4):553-7.

13. Seo JP, Jang SH. Characteristics of corticospinal tract area according to pontine level. Yonsei Med J. 2013;54(3):785-7.

14. Han BS, Hong JH, Hong C, et al. Location of the corticospinal tract at the corona radiata in human brain. Brain Res. 2010;1326:75-80.

15. Kwon HG, Choi BY, Chang CH, et al. Recovery of an injured corticospinal tract during a critical period in a patient with intracerebral hemorrhage. NeuroRehabilitation. 2013;32(1):27-32. 